\title{
Pensar a formação e a pesquisa na pós-graduação stricto sensu
}

\section{Presentation: To think the training and the research in the stricto sensu postgraduated}

\section{Presentación: Pensar la formación y la investigación en el posgrado stricto sensu}

\author{
Maria de Fatima Quintal de Freitas* \\ Jusamara Souza $^{* *}$
}

A proposta deste trabalho será desenvolvida em quatro seções com a perspectiva de que as informações possam subsidiar uma discussão a respeito da pesquisa e da formação na pós-graduação. Busca-se uma reflexão que considere as suas interfaces entre pesquisa e formação, visto que são mutuamente dependentes, ainda mais se entendermos que a pós-graduação deveria fornecer as ferramentas metodológicas e os embasamentos epistemológicos necessários para a constituição de quadros de pesquisadores-docentes de alto nível, comprometidos com a realidade do país e a produção de conhecimentos nacionais socialmente relevantes.

$\mathrm{Na}$ primeira seção serão apresentadas, de maneira breve, informações sobre as diretrizes centrais da pós-graduação, mostrando um rápido crescimento nacional e internacional. Na segunda seção, apresentar-se-ão interrogantes considerados centrais nessa reflexão sobre finalidade e razões da pós-graduação, assim como desafios e possibilidades, no tocante à sua tarefa de formar e qualificar para a pesquisa. $\mathrm{Na}$ terceira seção, reúnem-se aspectos que permitem identificar pontos críticos relacionados ao cotidiano do fazer e produzir pesquisa na pós-graduação, relacionando-os com o processo de formar pesquisadores

* Universidade Federal do Paraná. Programa de Pós-Graduação em Educação. Curitiba, Paraná, Brasil. E-mail: fquintal@terra.com.br. https://orcid.org/0000-0002-0414-199X

** Universidade Federal do Rio Grande do Sul. Programa de Pós-Graduação em Música. Porto Alegre, Rio Grande do Sul, Brasil. E-mail: jusa.ez@terra.com.br. https://orcid.org/00000001-6392-3951 
específicos e pontuais ou com visão e conhecimento de totalidade. Na quarta seção, apresentaremos os oitos artigos aqui reunidos, que podem fornecer alguns liames com relação à temática central deste dossiê: "Pesquisa $\mathrm{e}(\mathrm{m})$ formação na pós-graduação: desafios e práticas".

\section{Primeira seção: pós-graduação e suas metas}

Independentemente das metas específicas e das características socioeconômicas e políticas em que cada Plano Nacional da Pós-Graduação (PNPG) foi estabelecido, pode-se dizer que em todos eles a pós-graduação e seu sistema foram considerados como uma alavanca importante para políticas de Estado, internas e/ou externas, seja no ideário e composição de ações no âmbito das políticas públicas, seja na contabilização da formação de recursos humanos qualificados e publicizando os conhecimentos nacionais. Assim, desde a década de 1970, período em que a CAPES implantou a avaliação sistemática na pós-graduação, até meados da segunda década do segundo milênio, a produção da pós-graduação brasileira iniciou um processo de visibilidade para fora do país (cresceu mais de 165 vezes nos periódicos), embora ainda não se aproxime do ranking das publicações internacionais (KUENZER; MORAES, 2005).

Vários são os trabalhos que mostram que a pós-graduação stricto sensu é responsável pelo crescimento da produção científica no Brasil nos últimos 45 anos (OLIVEIRA; MORAES, 2016; KUENZER; MORAES, 2005; CATANI; OLIVEIRA; MICHELOTTO, 2010). Como exemplo temos o forte investimento que a CAPES fez no ano de 2003, quando havia no país 25.979 dissertações de mestrado e 8.094 teses de doutorado, nos 1.752 programas de pós-graduação existentes à época, dos quais $90 \%$ dos melhores encontravam-se nas instituições de ensino superior públicas. Nesse ano, esta agência de fomento à pesquisa fez um massivo investimento ( $81 \%$ do seu orçamento total - cerca de 450 milhões) em bolsas para capacitação, ao nível de doutorado e de mestrado, atingindo cerca de 106 mil pós-graduandos (BRASIL, 2005; KUENZER; MORAES, 2005).

Em todos os PNPG, direta ou indiretamente, a temática da formação e da qualidade constituem-se em pilares fundamentais do sistema da pós-graduação brasileira. O Sistema Nacional da Pós-Graduação (SNPG) estabelece duas metas centrais: formar competentes professores com qualidade e atender às necessidades do ensino, além de formar excelentes pesquisadores no campo das ciências básicas e das ciências aplicadas, cuja proposta era dobrar o número de doutores até o final da primeira década do segundo milênio (BRASIL, 2005, 2010). 


\section{Segunda seção: razões e possibilidades da pós-graduação}

Poderíamos começar esta breve reflexão sobre a formação na pós-graduação inserindo alguns eixos orientadores. O primeiro eixo reúne duas perguntas centrais a respeito da formação na pós-graduação: uma do ponto de vista institucional e outra da perspectiva pessoal. O segundo eixo propõe considerações sobre a realidade da pós-graduação identificando desafios e problemas enfrentados no que diz respeito à produção de conhecimentos e projetos assumidos junto à sociedade.

No tocante ao primeiro eixo, e tendo a universidade, a ciência e a sociedade como atores centrais, a primeira indagação de perspectiva institucional, seria: para que formamos mestres e doutores? E a questão de ordem pessoal, como atores individuais, seria: por que nós nos formamos como mestres e doutores?

Responder, a essas indagações, traz para o debate aspectos importantes relativos a:

- discutir que a relevância da titulação na pós-graduação não deveria se restringir à garantia da empregabilidade. Devido aos investimentos de alta qualidade feitos para esse tipo de formação, é esperado que haja algum retorno para a sociedade, através de melhores professores nos cursos de graduação, melhores especialistas e investigadores que contribuam para a continuidade e aprimoramento do conhecimento científico com significado coletivo e social;

- considerar se haveria profissões e/ou atividades que seriam mais apropriadas para receber pesquisadores doutores, recém-saídos da universidade, também é um aspecto relevante. Seria esperado que também nesses espaços, para além dos muros acadêmicos, houvesse um compromisso com a continuidade e multiplicação dos processos aprendidos para desenvolver e implementar pesquisas, estabelecendo fortes redes de cooperação entre universidade e sociedade, em que aquela teria preservado seu papel de autonomia e fomentadora dos processos de formação para a produção de conhecimentos através da pós-graduação stricto sensu.

Intimamente relacionada a essas considerações, a segunda indagação, na perspectiva dos atores individuais, aponta para o cotidiano do trabalho na pós-graduação. Esse cotidiano apoia-se nas relações orientador-orientando e na participação nos grupos de pesquisa, preocupando-se com a formação para a pesquisa e, consequentemente, para a produção de conhecimento. 
Tomando como base o crescimento dos programas de pós-graduação, traduzido pelo número de títulos de doutores que foram obtidos, poder-se-ia pensar em um continuum com dois polos distintos, quanto à finalidade e futuro da titulação obtida. Em um dos polos, estaria o fato de se titular (obtenção do título de doutor) para atuar em algum setor específico, externo à universidade, em que não necessariamente haveria continuidade do que foi aprendido e produzido durante os estudos no mestrado e doutorado. No outro polo, estaria o titular-se para ingressar no mundo da formação acadêmica, por meio da docência e da pesquisa em que seria dada continuidade ao processo vivido de aprendizagem e formação, contribuindo para a formação de novos quadros de pesquisadores e docentes. Localizar-se em um desses polos poderia estar na dependência, também, de alguns fatores que estiveram presentes durante o mestrado e doutorado, a saber:

- ter pertencido e participado de projetos contínuos de pesquisa, com regularidade e constância;

- ter participado em um coletivo orgânico (grupos de pesquisa) de pesquisadores que se encontravam em diferentes estágios dessa formação (iniciação científica, Programa de Educação Tutorial da Coordenação de Aperfeiçoamento de Pessoal de Nível Superior (PET/CAPES), outros), e por isso ter aprendido a desempenhar níveis variados de atividades científicas, individuais e coletivas.

Essas condições podem favorecer a construção de uma cultura da pesquisa e ciência, dentro dos programas de pós-graduação, que tivesse continuidade e que envolvesse em igual medida docentes, orientadores e pós-graduandos. Nessa cultura, a pesquisa adquiriria importância para a construção de redes de trabalho colaborativo, aliando-se ao processo de formação de pesquisadores que passem a se implicar também com a docência. E esta, por sua vez, fortalecer-se-ia ao poder contar com professores que se atualizem e se aproximem da realidade social, empregando a pesquisa e a produção de conhecimentos, também, como ferramentas pedagógicas.

\section{Terceira seção: condicionantes do trabalho na pós-graduação}

Formar mestres e doutores nos cursos de pós-graduação envolve uma complexidade de situações, fatores e circunstâncias, muitas vezes, pouco consideradas quando se fala do sistema da pós-graduação. As condições do trabalho realizado por ambos (orientadores e orientandos), os processos de aprendizagem 
envolvidos, as dificuldades e avanços, são todos aspectos importantes relacionados ao formar-se doutor e mestre, do ponto de vista do pós-graduando, e ao estar formando mestres e doutores, da perspectiva dos professores e orientadores na pós-graduação. Trata-se, também, de uma dinâmica complexa e cuja experiência na realização dos trabalhos imprime diferenças nos modos de enfrentamento e caminhos encontrados para resolver dificuldades e dar continuidade ao trabalho conjunto (MANCEBO, 2007; MILLS, 2009).

Ao longo dos estudos realizados na pós-graduação, alguns desses aspectos mostram-se delicados e, às vezes, decisivos. Além de trazerem questionamentos sobre os condicionantes (des)favoráveis a esse processo de formação, como passaremos a expor brevemente.

Um deles refere-se ao peso e lugar que a obtenção do título de doutor ocupa na construção do papel de pesquisador experiente, contribuindo para que domínios epistemológicos tenham sido incorporados e ajudem no desenvolvimento de projetos de pesquisa autônomos e independentes. Fazer esta análise permitiria identificar quais aspectos poderiam ser levados em conta para uma formação ampliada e continuada como pesquisador, mesmo quando do término dos estudos de pós-graduação; incluindo-se, por exemplo, os casos de estudos no âmbito de Pós-Doc. Embora sejam importantes, os estudos após o doutorado, não necessariamente garantem a continuidade da formação de pesquisadores, caracterizada pela aprendizagem de uma maior variação epistemológica.

Outro condicionante consiste na finalidade do trabalho colaborativo entre orientadores, orientandos e grupos de pesquisa. Os aspectos pedagógicos da aprendizagem e formação ligados ao se tornar pesquisador e aprender a orientar podem ser sistematizados, dentro, por exemplo, dos grupos de pesquisa e dos estágios de docência em ensino superior que vários programas de pós-graduação instituíram. Vários têm sido os relatos de pós-graduandos sobre as contribuições de participarem dessas atividades de maneira continuada e sistemática.

Um terceiro condicionante, derivado dos anteriores, remeteria à análise sobre a pesquisa e o conhecimento produzido, no tocante às razões de tal objeto investigado, à destinação dos resultados produzidos e à contribuição para um tipo de setor e/ou população. Falar disso remete à discussão sobre os seguintes aspectos: a) os impactos das teses e dissertações produzidas; b) o compromisso social e as implicações éticas da utilização e da participação de grupos e de setores da sociedade nas pesquisas realizadas; c) a formação na pós-graduação que não pode se justificar por uma necessidade de autoformação, no sentido dos mestrados e doutorados terem sido também procurados com a finalidade precípua de aprimoramento dos estudos.

E, por fim, um quarto condicionante, estaria no conjunto e dinâmica de atividades e disciplinas que acontecem durante os estudos de pós-graduação. 
O grau de consistência e interesse das disciplinas oferecidas, articulado ao objeto da tese e dissertação, de um lado, e o envolvimento e disponibilidade para dedicar-se aos estudos para além da especificidade do objeto de pesquisa, de outro, contribuem para ampliar horizontes epistemológicos. Isso, ao mesmo tempo, traz para os orientadores exigências maiores para que aprofundem os debates e produções, com o objetivo de acompanhar tais horizontes. Nesse cenário, as exigências de produtividade bibliográfica e prazos exíguos, por sua vez, criam pontos críticos entre a busca por aprofundamentos e ampliação de aprendizagens versus a agilidade e eficiência de produções.

Entretanto, a despeito de tais condicionantes serem (des)favoráveis à realização dos trabalhos na pós-graduação, tem se observado um crescimento significativo neste milênio, em especial se considerarmos que a pós-graduação brasileira iniciou-se nos anos 1960, com poucos programas e em poucas áreas de conhecimento e regiões do país.

\section{Quarta seção: diálogos entre os trabalhos}

A presente proposta tem como objetivo reunir artigos em torno do eixo temático "Formação e(m) Pesquisa na Pós-Graduação: práticas e desafios". Toma-se aqui como referência o fato de que a pós-graduação stricto sensu, no campo das ciências humanas e sociais, já vem se consolidando, em sua trajetória não só de desenvolvimento de pesquisa, como também voltada para a formação de investigadores para comporem e participarem, de modo consistente e regular, na produção de conhecimento relevante nas áreas em que foram formados.

Apesar disso, os desafios propostos para essa formação, assim como a análise sobre as variadas práticas que vêm sendo implementadas, em distintos programas de pós-graduação, nas últimas três ou quatro décadas, ainda têm tido pouca visibilidade no cenário das discussões científicas. Tais desafios referem-se a obstáculos presentes em vários campos disciplinares, cuja análise permitiria identificar dimensões que interferem no processo da investigação, assim como no da formação dentro da pós-graduação, tais como os relativos:

- às condições de trabalho ligadas à orientação e ao desenvolvimento de pesquisas;

- à criação e ao fortalecimento de grupos de investigação;

- aos processos relacionados à própria orientação como uma das etapas necessárias para a formação; 
- às decisões epistemológicas que afetam o desenvolvimento dos trabalhos de pesquisa durante o processo de se formar na pós-graduação e de se tornar pesquisador;

- aos trabalhos colaborativos nas diferentes etapas da formação durante o processo da pós-graduação;

- às influências favoráveis e desfavoráveis advindas de instâncias, processos e temas que interferem na obtenção de recursos a serem utilizados nos projetos de pesquisa; entre outros.

Buscou-se organizar o presente dossiê com a finalidade de reunir docentes investigadores, nacionais e estrangeiros, que atuam em Programas de Pós-Graduação, cuja prática como orientadores(as) de mestrado e/ou doutorado e, também, como professores-investigadores, tem sido marcada pelas peculiaridades e vicissitudes que cada um tem enfrentado e vivido em seu cotidiano de trabalho. Os programas de pós-graduação stricto sensu, nos quais atuam, pertencem às áreas da educação, educação musical, sociologia rural e psicologia, no Brasil [Pontifícia Universidade Católica de Campinas (PUCCAMP), Pontifícia Universidade Católica de São Paulo (PUC-SP), Universidade Federal do Paraná (UFPR), Universidade Federal do Rio Grande do Sul (UFRGS), Universidade do Estado da Bahia (UNEB)] e no exterior (Argentina, México e Portugal).

Reuniram-se artigos que expressam, também, a experiência dos autores na docência na pós-graduação e no exercício da orientação, guiado pela formação por intermédio da pesquisa. O recorte teórico-epistemológico adotado pelos(as) autores(as) fundamenta-se no campo de suas produções bibliográficas e das linhas de investigação na pós-graduação, referindo-se a relatos de pesquisa dirigidos à temática central neste dossiê: refletir sobre as práticas implementadas na interface entre pesquisa e formação na pós-graduação, assim como sobre o enfrentamento dos desafios e obstáculos oriundos de tais trabalhos.

O primeiro artigo do professor Dr. Antônio Dias Nascimento, intitulado "Formação em Pesquisa na Pós-Graduação: práticas e desafios. A formação do pesquisador em Educação na Universidade do Estado da Bahia" traz um breve panorama da criação da pós-graduação no Brasil, discutindo os reflexos para a região Nordeste. Faz uma análise sobre a formação do pesquisador no Programa de Pós-Graduação em Educação e Contemporaneidade da Universidade do Estado da Bahia, cujo mestrado foi autorizado em 1999 e o doutorado em 2008.

O professor Dr. Jorge Mario Flores Osorio traz no artigo "Retos y contradicciones de la formación de investigadores en México" uma análise das políticas de estado do México para as instituições de ensino superior quanto à formação de pesquisadores. Ocupa o cargo de diretor de doutoramento em Psicologia na Universidade de Tijuana e é Presidente/Pesquisador do Centro Latinoamericano 
de Investigação, Intervenção e Atenção Social, há vários anos no México. Com base em registros e em entrevistas participantes junto a docentes e pesquisadores de vários programas de pós-graduação em Educação e Psicologia, ele analisa o Conselho Nacional de Ciência e Tecnologia (CONACyT) no México (equivalente ao nosso $\mathrm{CNPq}$ ) quanto às funções atribuídas à investigação nos mestrados e nos doutorados.

No artigo "Perspectiva histórica y estado actual de la formación Doctoral en Psicología en Argentina: el Doctorado de la Universidad Nacional de Córdoba", os professores Drs. Silvina Brussino e Juan Carlos Godoy, do Programa Doutoral em Psicologia da Universidade de Córdoba, na Argentina, e membros do CONICET, realizam uma descrição e análise crítica da formação acadêmica em pesquisa ao nível do doutorado em Psicologia na Argentina. Investigam os impactos, no âmbito científico e acadêmico, dessa formação, analisando o estado atual de oferta de programas de doutorado em Psicología, tendo como fonte os informes do Consejo Nacional de Avaliação e Acreditação Universitária, com foco no Programa de Doutorado da Universidade Nacional de Córdoba.

No quarto artigo, do professor Dr. Salvador Antonio Mireles Sandoval, do Programa de Pós-Graduação em Psicologia Social da PUC-SP, intitulado "Formação em Métodos de Pesquisa na Pós-Graduação: abordagens multimétodos para as demandas da atualidade", encontra-se uma análise da articulação entre conhecimento produzido na universidade e utilização por programas de governo. Analisa os aspectos da formação em pesquisa que se mostram importantes para que os resultados de estudos científicos empíricos sejam incorporados em políticas públicas de ação governamental, destacando para isso a necessidade de aprendizagem e domínio de variados métodos de pesquisa.

No artigo "O acervo Helena Antipoff como laboratório de pesquisa sobre a história das Ciências da Educação", da professora Dra. Regina Helena de Freitas Campos, do Programa de Pós-Graduação em Educação da Universidade Federal de Minas Gerais (UFMG), encontramos uma descrição, registro e análise histórica sobre as atividades de pesquisa que foram desenvolvidas, tomando como base o acervo documental do Centro de Documentação e Pesquisa Helena Antipoff, psicóloga e educadora russo-brasileira. Esta instituição foi criada em 1980 e teve o apoio dos trabalhos de pesquisa documental e recuperação da memória histórica, descritos no artigo, mostrando sua contribuição à formação em pesquisa na pós-graduação em psicologia, educação e história da ciência.

As professoras Dras. Amélia Lopes e Isabel Menezes, do Programa Doutoral em Educação e Psicologia da Universidade do Porto, em Portugal, em seu artigo "A construção de si como investigador: reflexões sobre os processos 
de formação pós-graduada", tecem análises sobre o processo de formação na pós-graduação stricto sensu. Com base em dois estudos empíricos realizados junto aos pós-graduandos da Universidade do Porto, somados à experiência e registros das autoras como diretoras no Programa de Pós-Graduação em Ciências da Educação, analisam experiências que se tornaram importantes para o processo de construção dos pós-graduandos como pesquisadores durante os cursos.

No penúltimo artigo, "Formação em pesquisa na pós-graduação: possibilidades e desafios a partir da orientação", as organizadoras deste dossiê, professoras Dras. Maria de Fatima Quintal de Freitas e Jusamara Souza, respectivamente, dos Programas de Pós-Graduação em Educação da UFPR e em Música da UFRGS, analisam a relação entre as condições objetivas e subjetivas do trabalho na pós-graduação e os impactos na formação dos pós-graduandos, por meio da pesquisa. Sistematizaram e analisaram registros e relatos obtidos nos processos interacionais de orientações realizadas em programas de pós-graduação em educação, educação musical e psicologia, no período de 1995 a 2017. Como parte da análise, identificam alguns efeitos psicossociais que podem interferir na produção de conhecimento, na qualidade das interações, nas orientações e na formação de pesquisadores.

O dossiê termina com o artigo "Pesquisa \& Mudança Social: desafios e dificuldades para a formação em psicologia”, da professora Dra. Raquel Souza Lobo Guzzo, do Programa de Pós-Graduação em Psicologia da PUCCAMP. A pesquisadora discorre sobre as dificuldades e desafios que são enfrentados no processo de formação de pesquisadores na área da Psicologia no Brasil. Analisa os aspectos epistemológicos que sofreram influência dos condicionantes históricos e sociais, que criaram diretrizes para as políticas de formação na pós-graduação e a preparação em pesquisa e formação em psicologia.

No campo das ciências humanas e sociais, a Pós-Graduação stricto sensu no Brasil, cada vez mais vem se fortalecendo, também por intermédio de sistemas e plataformas de avaliação, atualização e divulgação do conhecimento. Entretanto, tomando-se como referência as peculiaridades e desafios da formação com base na pesquisa na pós-graduação, pode-se dizer que as discussões a respeito desta intrincada relação, na ótica dos processos internos da pós-graduação, ainda são tímidas. Espera-se que este dossiê possa se situar no cenário dos debates científicos, atuais e necessários, relativos ao trabalho e ao ofício do tornar-se orientador(a), docente e investigador na pós-graduação, no campo das ciências humanas e sociais, comprometido com a formação em pesquisas nesse âmbito. 


\section{REFERÊNCIAS}

CATANI, A. F.; OLIVEIRA, J. F.; MICHELOTTO, R. M. As políticas de expansão da educação superior no Brasil e a produção do conhecimento. Série-Estudos - Periódico do Programa de Pós-Graduação em Educação da UCDB. Campo Grande-MS, n. 30, p. 267-281, jul./dez. 2010.

KUENZER, A.; MORAES, M. C. M. Temas e tramas na pós-graduação em educação. Educação \& Sociedade, Campinas, v. 26, n. 93, p. 1341-1362, set./dez. 2005.

MANCEBO, D. Trabalho docente: subjetividade, sobre implicação e prazer. Psicologia: Reflexão e Crítica, v. 20, n. 1, p. 74-80, 2007.

MILLS, C. Wright. Sobre o artesanato intelectual e outros ensaios. Trad. Maria Luiza X. de A. Borges. Rio de Janeiro, Zahar, 2009.

OLIVEIRA, J. F.; MORAES, K. N. Produção do conhecimento na universidade pública no Brasil: tensões, tendências e desafios. Educação em Revista, Belo Horizonte, v. 32, n. 4, p. 73-95, out./dez. 2016. 